\title{
A SURVEY OF CIIDAE (COLEOPTERA, TENEBRIONOIDEA) OF THE HYRCANIAN FOREST (IRAN) WITH NEW FAUNISTIC RECORDS
}

\author{
S. Amini ${ }^{1,3}$, R. Krolik ${ }^{2}$, J. Nozari ${ }^{1 *}$, M. E. Farashiani ${ }^{3}$ \& F. Kazerani ${ }^{3}$ \\ ${ }^{1}$ Department of Plant Protection, College of Agriculture and Natural Resources, \\ University of Tehran, Karaj, Iran.E-mail:sudabe.amini@ut.ac.ir \\ ${ }^{2}$ Mickiewicza, 8, 46-200 Kluczbork, Poland \\ E-mail: agrilus@poczta.onet.pl \\ ${ }^{3}$ Research institute of Forest and Rangeland, Agriculture Research, \\ Education and Extension Organization (AREEO), Tehran, Iran \\ E-mail: efarashini@gmail.com, farzane.kazerani@gmail.com \\ E-mail:nozari@ut.ac.ir \\ ${ }^{*}$ Corresponding author
}

\begin{abstract}
A Survey of Ciidae (Coleoptera, Tenebrionoidea) of the Hyrcanian Forest (Iran) with New Faunistic Records. Amini, S., Krolik, R., Nozari, J., Ebrahim Farashiani, M., Kazerani, F. - Ciidae are a small family of mycetophagous beetles with only fifteen species so far recorded in Iran. The occurrence of nine of them has now been confirmed. Additional 8 species belonging to 5 genera collected during the 20142017 survey in the Hyrcanian Forest, North Iran, are recorded for the first time in Iran. Four species are excluded form the Iranian fauna. As a result of this study, the number of species known from Iran has increased to 19 .
\end{abstract}

Key words: Ciid beetles, new records, fauna, forest, fungi, Iran, Middle East.

\section{Introduction}

Ciidae Leach, 1819 is a cosmopolitan family of minute tree-fungus beetles comprising more than 700 described species in 51 genera worldwide (Lawrence, 2016, 2019; Souza-Gonçalves et al., 2018). There have been only limited taxonomic studies of this family with only fifteen species so far recorded from Iran (Jelínek, 2008; Amini et al., 2014; Królik, 2016; Lopes-Andrade et al., 2016, Samin et al., 2018 a, b). Ciidae are tiny beetles, less than $7 \mathrm{~mm}$ in size, with oval to elongated bodies (Lawrence \& Lopes-Andrade, 2010). Mycophagy is predominant in the family. All stages of most species are completely dependent on the fungus for life aspects such as food, shelter, and breeding and usually live inside basidiomes, on the surface the basidiocarps of tree fungus or under the bark of dead trees in the mycelial layer (Orledge \& Reynolds, 2005; Graf-Peters et al., 2011). 
Ciidae species are also found under the bark of dead and weakened twigs which are nearly similar to bark beetles habitants (Kawanabe, 1995). A few ciid species have been recorded as introduced pests of commercially dried fungi, such as Cis chinensis Lawrence, 1991 and Cis asiaticus Lawrence, 1991 (Lawrence \& Lopes-Andrade, 2010).

The Iranian coast of the Caspian Sea is dominated by the Caspian Hyrcanian Mixed Forest ecoregion, with a total area of 1.85 million ha. The Hyrcanian Forest is a green belt of temperate deciduous forests located on the northern slopes of the Alborz Mountain and southern borders of the Caspian Sea. The Hyrcanian Forest includes three provinces of Iran (Guilan, Mazandaran, and Golestan) from Astara in the northwest to the vicinity of Gorgan (Golidaghi) in the northeast of Iran (Sagheb Talebi et al., 2014). Hyrcanian forests are marked by a high diversity of host plants and a humid climate, which is a suitable habit for saproxylic beetles. The purpose of this study was to investigate the Iranian fauna of Ciidae and provide new records of species in North forests of the country.

\section{Material and methods}

This study was done during 2013-2017 in various areas of Iran's Hyrcanian Forest. All the specimens cited in this paper were collected on weakened trees by hand collecting and using flight intercept traps. Traps included water and propylene glycol in $1: 1$ portion as an insect preservation, and were located 50 meters apart. Specimens were also collected from beneath the bark of twigs, then moved to absolute alcohol for further preservation. The information on each collection site was registered and showed on Map (fig. 2). Additional material was reared from the fungi collected during the survey. In this study, we also used data from specimens deposited at the Museum of Natural History, Wrocław University, Poland, which were collected in the 1960s by Prof. Dr. Hab. Andrzej Warchałowski, a world-renowned specialist in Chrysomelidae (Coleoptera). Among species new to Iranian fauna or recently recorded, six species with quite characteristic morphology are illustrated (figs 1, 1-6). The pictures were taken using a Nikon D7500 camera and the Nikon M Plan10 lens. Images stacking was performed using Helicon Focus 7 (license holder is the second author). Species identification is based on Reitter (1901), Lohse (1967), Lawrence (1991) and Drogvalenko (2002). Male genital preparations were made as necessary for the accurate identification of certain ciid species.

The specimens here cited are deposited in the following collections:

MNHW - Museum of Natural History, Wrocław University, Wrocław, Poland.

RIFR - Research Institute of Forests and Rangelands, Tehran, Iran.

RKPC - Roman Królik's private collection, Kluczbork, Poland.

USMB - Upper Silesian Museum, Bytom, Poland.

ZMUT - Zoology Museum of Tehran University.

New records from the Iranian fauna were marked by an asterisk $\left(^{*}\right)$.

\section{Results}

During this study a total of 937 Ciidae of 7 genera and 17 species were collected and identified. Of the species already recorded from Iran only Cis boleti, C. castaneus, C. chinensis, Strigocis bicornis and Sulcacis nitidus were collected in the study area. Cis comptus, C. fusciclavis, C. reitteri, C. rugulosus, C. striatulus, C. submicans, C. tomentosus, Ennearthron cornutum, Octotemnus rugosopunctatus, Ropalodontus perrini, Sulcacis fronticornis, Xylographus bostrichoides are recorded for the first time in the fauna of Iran.

\section{List of species}

\section{Cis boleti (Scopoli, 1763)}

Material examined: Guilan Prov., Rasht, Lakan, $37^{\circ} 09.6^{\prime}$ N, 49³4.5' E, 06.2006.

Species inhabiting almost the whole Palearctic Region.

Distribution: Algeria, Azerbaijan, Austria, Belgium, Bosnia and Herzegovina, China (Sichuan), Belarus, Croatia, Russia (European Territory, Far East), Czech Republic, Denmark, Estonia, Finland, France, Great Britain, Germany, Georgia, Hungary, Iran, Ireland, Italy, Japan, Latvia, Liechtenstein, Lithuania, Mongolia, Netherland, Norway, Poland, Romania, Slovakia, Slovenia, Spain, Sweden, Switzerland, Ukraine (Jelínek, 2008). 


\section{Cis castaneus (Herbst, 1793)}

Material examined: Golestan Prov., 45 km NE of Minudasht, 37²3.104' N 5550.012' E, $584 \mathrm{~m}$ amsl, 12.06.2015, 1 ex. leg. R. Królik (RKPC); 11 km SE of Minudasht, 37010.101' N 55²8.211' E, 447 m amsl, $13-$ 15.06.2015, 15 exx. leg. R. Królik, from fungus on Populus L., 4 exx. reared 20.VI-5 07.2015, cult. R. Królik

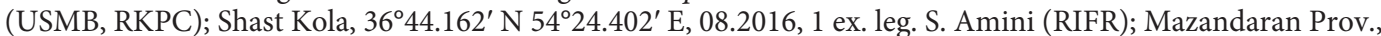
Chalus, $36^{\circ} 39.102^{\prime} \mathrm{N}^{\circ} 1^{\circ} 25.225^{\prime} \mathrm{E}, 14-19.02 .1966,46$ exx. reared 17.05.1966-4.10.1968 leg. A. Warchałowski, cult. M. Kak (MNHW).

Distribution in Iran: Isfahan Prov., Natanz (Samin et al., 2018).

Species occurring from Scandinavia to mountain and foothill areas in Southern Europe; recorded also in the Russian Far East. Polyphagous species developing in various fungi species with hard basidiomes (most commonly Fomes (Fr.) Fr. and Ganoderma P. Karst.), growing mainly on deciduous trees.

Distribution: Azerbaijan, Austria, Belgium, Bosnia and Herzegovina, Belarus, Croatia, Russia (European Territory, Far East), Czech Republic, Denmark, Estonia, Finland, France, Great Britain, Germany, Greece, Hungary, Ireland, Italy, Liechtenstein, Lithuania, Netherland, Norway, Poland, Romania, Serbia and Montenegro, Slovakia, Slovenia, Sweden, Switzerland, Ukraine, (Jelínek, 2008), Spain (Diéguez Fernández, 2013), Iran (Samin et al., 2018).

Cis chinensis Lawrence, 1991 (fig. 1, 1)

Material examined: Mazandaran Prov., Sisangan National Park, 36³5.154' N 51 $48.251^{\prime}$ E, 8.06.2015, 2 exx. leg. T. Jaworski (RKPC).

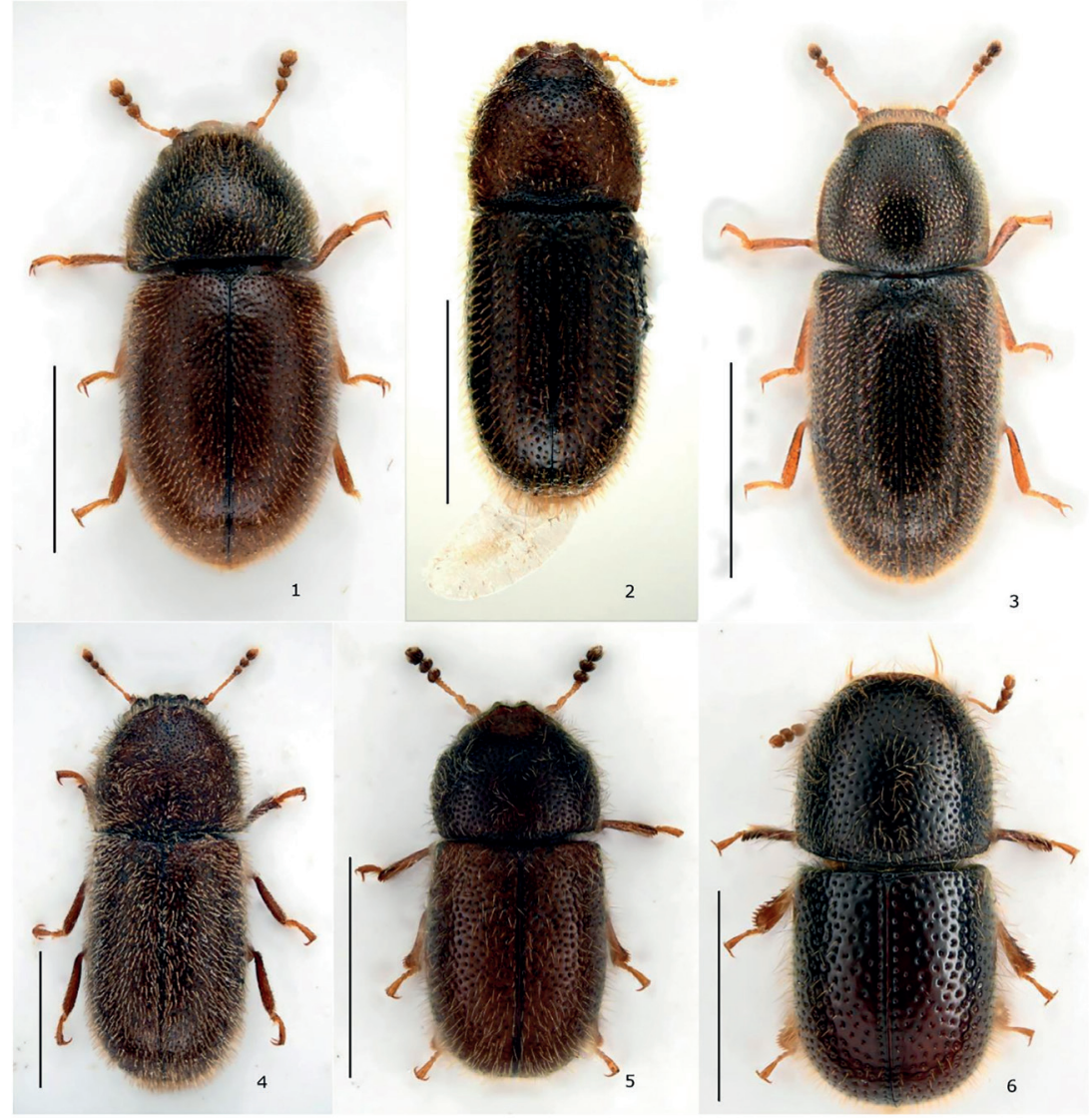

Fig. 1. 1 - Cis chinensis, Mazandaran Prov., Sisangan National Park; 2 - Cis reitteri, Golestan Prov., 11 km SE of Minudasht; 3 - Cis striatulus, Golestan Prov., $11 \mathrm{~km}$ SE of Minudasht; 4 - Cis tomentosus, Golestan Prov., $45 \mathrm{~km}$ NE of Minudasht; 5 - Ropalodontus perrini, Golestan Prov., $11 \mathrm{~km}$ SE of Minudasht; 6 - Xylographus bostrichoides, Mazandaran Prov., Sisangan National Park. Scale bars: $1 \mathrm{~mm}$. 
Distribution in Iran: Guilan Prov. (Amini et al., 2014, 2015).

Distribution in Palearctic: China(Lawrence, 1991), France (Rose, 2009), Germany (Lohse \& Reibnitz, 1991), Hungary (Reibnitz \& Kunz, 2011), Malta (Lopes-Andrade, 2008), Spain (Diéguez Fernández, 2013), Iraq (Shugran et al., 2018). Report from Iran about Cis multidentatus (Pic, 1917) (Amini et al., 2014) should be referred to Cis chinensis. The current list of host fungi is provided by Souza-Gonçalves \& Lopes-Andrade (2018).

${ }^{*}$ Cis comptus Gyllenhal, 1827

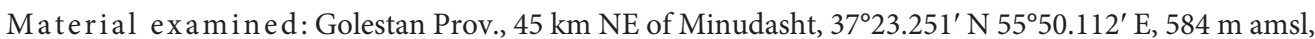
12.06.2015, 26 exx. leg. R. Królik et A. Lasoń (USMB, RKPC); $11 \mathrm{~km} \mathrm{SE} \mathrm{of} \mathrm{Minudasht,} 37^{\circ} 10.256^{\prime} \mathrm{N} 55^{\circ} 28.301^{\prime} \mathrm{E}$, 447 m amsl, 13-15.06.2015, 20 exx. leg. R. Królik et A. Lasoń, from fungus on Populus sp. (USMB, RKPC); Mazandaran Prov., Chalus, 36³9.102' N 51²5.153' E, 14-19.02.1966, 2 exx. reared 17.05.1966-4.10.1968 leg. A. Warchałowski, cult. M. Kak (MNHW); 28 km E of Marzanabad, 36²6' N 51 $32^{\prime}$ E, 20.05.2017, 30 exx. leg. A. Lasoń (USMB, RKPC).

Widespread Palearctic species. It inhabits almost the whole of Europe, known from North Africa, reaches its range to the Far East. It develops on the soft basidiomes of fungi (especially Bjerkandera P. Karst., Coriolopsis Murrill and Trametes Fr.) on deciduous trees.

Distribution: Azerbaijan, Armenia, Austria, Belgium, Bosnia and Herzegovina, Bulgaria, Belarus, Croatia, Russia, Czech Republic, Denmark, Estonia, Finland, France, Germany, Georgia, Greece, Hungary, Italy, Kazakhstan, Macedonia, Morocco, Netherland, Norway, Poland, Romania, Slovakia, Spain, Sweden, Switzerland, Tunisia, Turkey, Turkmenistan, Ukraine (Jelínek, 2008). Iran (first record).

${ }^{\star}$ Cis fusciclavis Nyholm, 1953

Material examined: Golestan Prov., Ali-abade-Katoul, 36²43.152' N 54³9.142' E, 08.2016., 1 ex. leg. S. Amini (ZMUT).

It has been shown so far from Europe, from France to the central areas of the European part of Russia, to the north reaching Scandinavia and south to Italy. As host fungi were most often shown: Bjerkandera adusta (Willd.) P. Karst., Fomes fomentarius (L.) Fr. and Piptoporus betulinus (Bull.) P. Karst. (Reibnitz, 1999). It is also found under the loose bark of deciduous trees.

Distribution: Austria, Bosnia and Herzegovina, Belarus, Russia (Central European Territory), Czech Republic, Denmark, Finland, France, Germany, Italy, Netherland, Norway, Poland, Slovakia, Slovenia (Jelínek, 2008), Switzerland (Reibnitz et al., 2013). Iran (first record).

\section{Cis lasoni Królik, 2016}

Distribution in Iran: Golestān Prov., 11 km SE of Minudasht (Królik, 2016).

Recently described species. Host fungi unknown.

Distribution: Azerbaijan, Iran (Królik, 2016).

\section{Cis lineatocribratus Mellié, 1849}

Distribution in Iran: Fars Prov., Kazerun (Samin et al., 2018).

It occurs in similar environments as $C$. castaneus. but is much less common.

Distribution: Azerbaijan, Austria, Bosnia and Herzegovina, Bulgaria, Belarus, Croatia, Russia (North and Central European Territory), Czech Republic, Denmark, Estonia, Finland, France, Germany, Georgia, Hungary, Italy, Latvia, Liechtenstein, Norway, Poland, Romania, Serbia and Montenegro, Slovakia, Sweden, Switzerland, Ukraine (Jelínek, 2008), Iran (Samin et al., 2018). 


\section{Cis matchanus Reitter, 1915}

Distribution in Iran: Mazandaran Prov., Chalus (Królik, 2016).

Rare species, often misidentified as the other species from the Cis castaneus species group. Its biology is as in most species of this group.

Distribution: Finland, Germany, Norway, Poland, Russia (North and South European Territory) (Jelínek, 2008), Bosnia and Herzegovina, Czech Republic, Georgia, Italy, Iran, Turkey, Ukraine, (Królik, 2016).

${ }^{\star}$ Cis reitteri Lopes-Andrade, 2002 (fig. 1, 2)

Material examined: Golestan Prov., $11 \mathrm{~km}$ SE of Minudasht, 370.265' N 55²8.102' E, $447 \mathrm{~m}$ amsl, 15.06.2015, 3 exx. Reared from fungus on Populus sp., 20.06-5.07.2015, leg. R. Królik (RKPC); Ali-abade-Katoul, $36^{\circ} 43.152^{\prime} \mathrm{N} 54^{\circ} 39.142^{\prime} \mathrm{E}, 08.2016 .9$ exx. leg. S. Amini (ZMUT); Mazandaran Prov., Chalus, $36^{\circ} 39.123^{\prime} \mathrm{N} 51^{\circ} 25.156^{\prime} \mathrm{E}$, 19.02.1966, 1 ex. leg. A. Warchałowski (MNHW).

The species is known so far from the Talysh Mountains in Azerbaijan, from where it was described by Reitter in 1884 under the name Cis setifer. The host fungus is unknown.

Distribution: Azerbaijan (Jelínek, 2008). Iran (first record).

\section{${ }^{\star}$ Cis rugulosus Mellié, 1849}

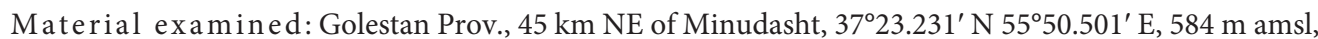

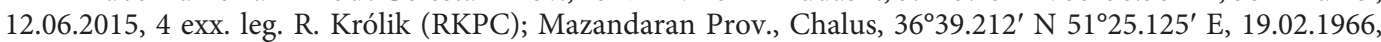
18 exx. reared 17.05.1966-4.10.1968 leg. A. Warchałowski, cult. M. Kak (MNHW); 13-14.02.1968, 7 exx. from fungus on Fagus orientalis Lipsky, leg. A. Warchałowski (MNHW).

Listed from most of Europe, it seems to be more common in its southern part. Certainly wider dispread in the Palearctic. It prefers for its development the soft basidiomes of the Trametes-type fungi growing on deciduous wood.

Distribution: Austria, Belgium, Russia (Central and South European Territory), Czech Republic, Estonia, Finland, France, Germany, Italy, Lithuania, Netherland, Slovakia, Sweden, Switzerland (Jelínek, 2008). Iran (first record).

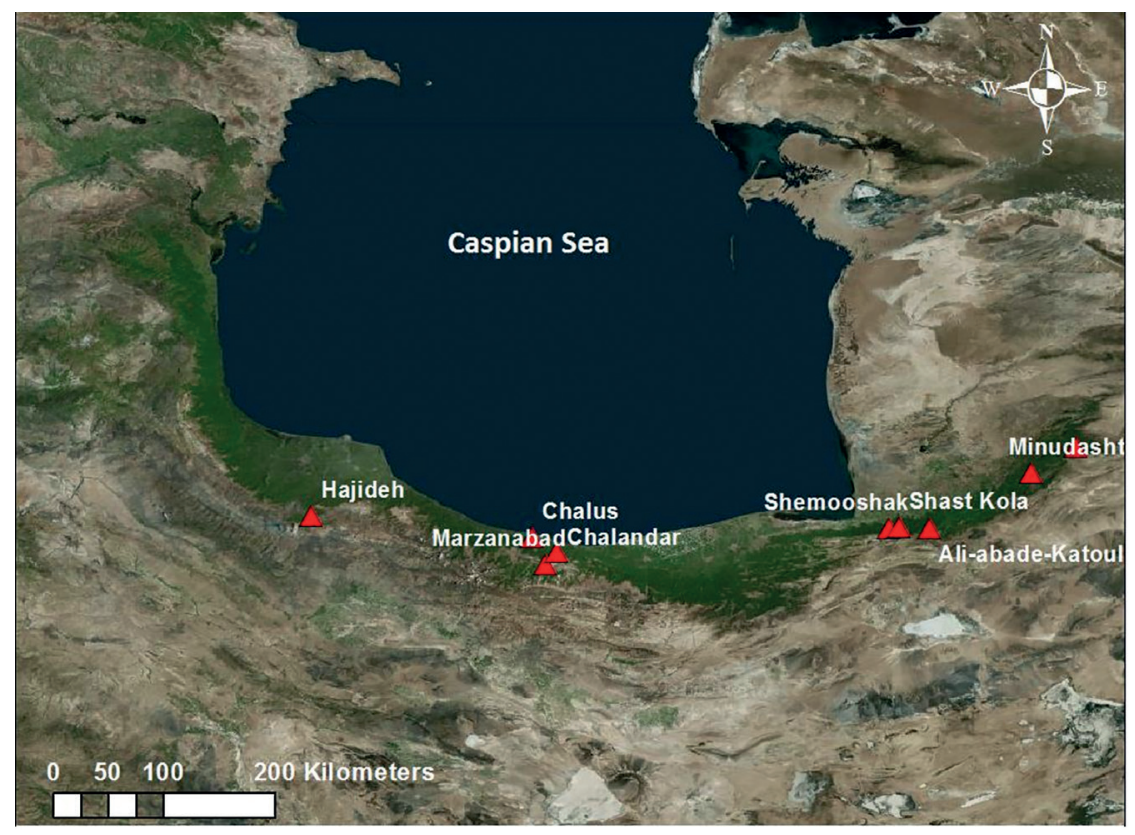

Fig. 2. Collecting sites in North forest of Iran. 
${ }^{\star}$ Cis striatulus Mellié, 1849 (fig. 1, 3)

Material examined: Golestan Prov., $45 \mathrm{~km} \mathrm{NE}$ of Minudasht, $37^{\circ} 23^{\prime} \mathrm{N} 55^{\circ} 50^{\prime} \mathrm{E}, 584 \mathrm{~m}$ amsl, 12.06.2015, 3 exx. leg. R. Królik (RKPC); 11 km SE of Minudasht, $37^{\circ} 10^{\prime} \mathrm{N} 55^{\circ} 28^{\prime}$, E $447 \mathrm{~m}$ amsl, 13-15.06.2015, 18 exx. leg. R. Królik et A. Lasoń, from various fungi (RKPC); Mazandaran Prov., Chalus, 36³9. N 5125. E, 19.02.1966, 25 exx. leg. A. Warchałowski (MNHW).

Species occurring locally in Europe, North Africa, Asia Minor and the Caucasus. Lives in the basidiomes of fungi of the genus Radulomyces M.P. Christ., mainly on deciduous trees (Reibnitz, 1999), less often coniferous.

Distribution: Algeria, Azerbaijan, Austria, Bosnia and Herzegovina, Croatia, France, Germany, Georgia, Greece, Hungary, Italy, Poland, Spain, Russia (South European Territory), Switzerland, Turkey (Jelínek, 2008). Iran (first record).

${ }^{*}$ Cis submicans Abeille de Perrin, 1874

Material examined: Guilan Prov., Hajideh env., 36²49.114' N 49³8.131' E, $872 \mathrm{~m}$ amsl, 8.06.2015, 1 ex. leg. A. Lasoń, (RKPC); Golestan Prov., $11 \mathrm{~km} \mathrm{SE}$ of Minudasht $37^{\circ} 10.101^{\prime} \mathrm{N} 55^{\circ} 28.211^{\prime} \mathrm{E}, 447 \mathrm{~m}$ amsl, 15.06.2015, 4 exx. leg. R. Królik, from fungus on Populus sp. (RKPC); Mazandaran Prov., Chalus, $36^{\circ} 39.561^{\prime}$ N $51^{\circ} 25.140^{\prime}$ E, 19.02.1966, 188 exx. reared 17.05.1966-4.10.1968 leg. A. Warchałowski, cult. M. Kak (MNHW); 13-14.02.1968, 4 exx. from fungus on Fagus orientalis leg. A. Warchałowski (MNHW).

It develops in basidiomes of various species of fungi of the genus Trametes, growing on deciduous trees.

Distribution: Azerbaijan, Armenia, Austria, Belgium, Bosnia and Herzegovina, Belarus, Croatia, Russia (European Territory, Far East), Czech Republic, Denmark, Estonia, Finland, France, Great Britain, Germany, Georgia, Greece, Hungary, Italy, Latvia, Lithuania, Netherland, Norway, Poland, Slovakia, Sweden, Switzerland, Turkey, Ukraine (Jelínek, 2008). Iran (first record).

${ }^{\star}$ Cis tomentosus Mellié, 1849 (fig. 1, 4)

Material examined: Golestan Prov., Ali-abade-Katoul, 36²43.152' N 54³9.142' E, 08.2016, 1 ex.

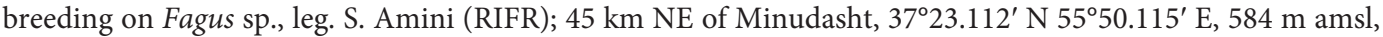
12.06.2015, 65 exx. leg. R. Królik et A. Lasoń, from small hubs growing on deciduous tree trunks, mainly Alnus Mill. (USMB, RKPC).

Listed from the southern part of Europe, North Africa and the Caucasus. The determination of host fungi species requires further research.

Distribution: Azerbaijan, Armenia, Bosnia and Herzegovina, Croatia, France, Georgia, Greece, Italy, Morocco, Romania, Russia (South European Territory), Serbia and Montenegro, Spain (Jelínek, 2008). Iran (first record).

Cis villosulus (Marsham, 1802)

Distribution in Iran: Guilan Prov., Siahkal (Samin et al., 2018).

This species prefers the mountain and foothill regions, it develops in Trametes species as T. hirsuta (Wulfen) Pilát, T. gibbosa (Pers.) Fr., T. suaveolens (L.) Fr., furthermore exhibited with Lenzites betulinus (L.) Fr., sporadically develops in Bjerkandera fumosa (Pers.) P. Karst. (Reibnitz, 1999).

Distribution: Austria, Belgium, Belarus, Croatia, Russia (European Territory, East Siberia, Far East), Czech Republic, Denmark, France, Great Britain, Germany, Georgia, Greece, Hungary, Ireland, Italy, Liechtenstein, Netherland, Norway, Poland, Portugal, Slovakia, Spain, Sweden, Switzerland, Turkey, Ukraine (Jelínek, 2008), Iran (Samin et al., 2018).

${ }^{\star}$ Ennearthron cornutum (Gyllenhal, 1827)

Material examined: Mazandaran Prov., Chalus, 36³9.441' N 51²5.119' E, 19.02.1966, 3 exx. reared 17.05.1966-4.10.1968, leg. A. Warchałowski, cult. M. Kak (MNHW). 
Species known from almost all of Europe, the Caucasus and some localities on Far East. Its development takes place in many different hubs growing on dead hardwood. The most frequently mentioned species are: Bjerkandera adusta, Daedalea quercina (L.) Pers., Fomes fomentarius, Fomitopsis pinicola (Sw.) P. Karst., Piptoporus betulinus, Schizopora Velen.

Distribution: Azerbaijan, Austria, Belgium, Bosnia and Herzegovina, China (Jilin), Croatia, Russia (European Territory), Czech Republic, Denmark, Estonia, Finland, France, Great Britain, Germany, Georgia, Hungary, Ireland, Italy, Japan, Liechtenstein, Lithuania, Netherland, Norway, Poland, Romania, Slovakia, Slovenia, Spain, Sweden, Switzerland, Ukraine (Jelínek, 2008). Iran (first record).

${ }^{\star}$ Octotemnus rugosopunctatus Drogvalenko, 2002

Material examined: Mazandaran Prov., Chalus, 36³9.102' N 51 ${ }^{\circ} 25.512^{\prime}$ E, 19.02.1966, 29 exx. reared 17.05.1966-4.10.1968, leg. A. Warchałowski, cult. M. Kak (MNHW); 13-14.02.1968, 2 exx. from fungus on Fagus orientalis leg. A. Warchałowski (MNHW).

A species described from the Crimean Peninsula, where it occurs in deciduous and mixed forests growing up to $1000 \mathrm{~m}$ amsl. It develops in the sporocarps of the fungi, mainly from the genus Trametes: T. suaveolens, T. versicolor (L.) Lloyd, T. pubescens (Schumach.) Pilát and Cerrena unicolor (Bull.) Murrill (Drogvalenko 2002). This find clearly expands the range of this species.

Distribution: Ukraine (Jelínek, 2008). Iran (first record).

\section{${ }^{\star}$ Ropalodontus perrini Reitter, 1878 (fig. 1, 5)}

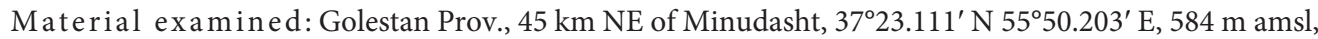
12.06.2015, 151 exx. leg. R. Królik et A. Lasoń, from various fungi (USMB, RKPC); $11 \mathrm{~km}$ SE of Minudasht, $37^{\circ} 10.254^{\prime} \mathrm{N} 55^{\circ} 28.102^{\prime}$ E, $447 \mathrm{~m}$ amsl, 13-15.06.2015, 22 exx. leg. R. Królik et A. Lasoń from fungus on Populus

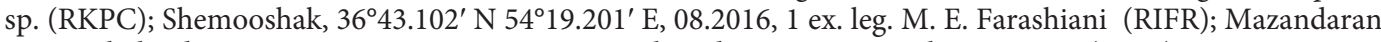
Prov., Chalandar, $36^{\circ} 32^{\prime} \mathrm{N} 51^{\circ} 37^{\prime}$ E, 08.2015, 5 exx. breeding on Acer sp., leg S. Amini (RIFR).

Species known so far from Azerbaijan and Georgia. Like most species of this genus, it develop in the hard sporocarps of the Fomes-type, growing on deciduous trees.

Distribution: Azerbaijan, Georgia (Jelínek, 2008). Iran (first record).

\section{Strigocis bicornis (Mellié, 1849)}

Material examined: Golestan Prov., 45 km NE of Minudasht, 37²3.102' N 5550.221' E, $584 \mathrm{~m}$ amsl, 12.06.2015, 3 exx. leg. R. Królik et A. Lasoń, from various fungi (RKPC); Mazandaran Prov., Chalus, 36 39.154' N 512ㄴ.168' E, 19.02.1966, 37 exx. leg. A. Warchałowski (MNHW); 13-14.02.1968, 8 exx. from fungus on Fagus orientalis leg. A. Warchałowski (MNHW).

Distribution in Iran: East Azarbaijan Prov., Bonab (Samin et al., 2018).

Species known so far from Europe (without its northern part), from North Africa and the Caucasus. Occurs in hubs of the genus Trametes. Usually coexist with the species of the genus Sulcacis Dury, 1917.

Distribution: Algeria, Azerbaijan, Bosnia and Herzegovina, Croatia, Czech Republic, France, Great Britain, Germany, Hungary, Italy, Romania, Spain, Switzerland, Ukraine (Jelínek, 2008), Iran (Samin et al., 2018), Poland (Królik et al., 2017).

\section{${ }^{\star}$ Sulcacis fronticornis (Panzer, 1805)}

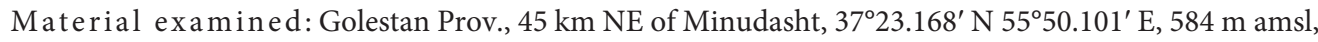
12.06.2015, 11 exx. leg. R. Królik et A. Lasoń, from various fungi (RKPC); $11 \mathrm{~km} \mathrm{SE}$ of Minudasht, $37^{\circ} 10.151^{\prime} \mathrm{N}$ 552․ 263' E, 447 m amsl, 13-15.06.2015, 68 exx. leg. R. Królik et A. Lasoń from fungus on Populus sp. (RKPC); Mazandaran Prov., Chalus, 36³9' N 51 $25^{\prime}$ E, 19.02.1966, 16 exx. leg. A. Warchałowski (MNHW). 
West Palaearctic species, more often found in the southern part of this area. Usually occurs in hubs of Trametes sp., Coriolopsis trogii (Berk.) Domański and Lenzites betulina (L.) Fr. growing on dead wood of deciduous trees.

Distribution: Azerbaijan, Austria, Belgium, Bosnia and Herzegovina, Belarus, Croatia, Russia (European Territory), Czech Republic, Denmark, Finland, France, Germany, Greece, Hungary, Italy, Latvia, Netherland, Norway, Poland, Romania, Serbia and Montenegro, Slovakia, Spain, Sweden, Switzerland, Ukraine (Jelínek, 2008). Iran (first record).

\section{Sulcacis nitidus (Fabricius, 1792)}

Material examined: Gilan Prov., Hajideh env., 3649.124' N 49³8.301' E, $872 \mathrm{~m}$ amsl, 8.06.2015, 1 ex. leg. A. Lasoń, (RKPC); Golestan Prov., 45 km NE of Minudasht, $37^{\circ} 23^{\prime}$ N 5550’ E, $584 \mathrm{~m}$ amsl, 12.06.2015, 1 ex.

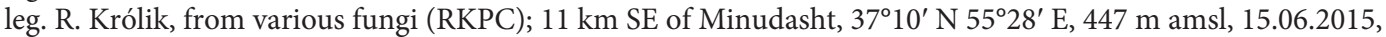
2 exx. leg. A. Lasoń, from fungus on Populus sp. (RKPC); Mazandaran Prov., Chalus, $36^{\circ} 39.121^{\prime}$ N 51 ${ }^{\circ} 25.514^{\prime}$ E, 19.02.1966, 10 exx. leg. A. Warchałowski (MNHW).

Distribution in Iran: Yazd Prov., Ardakan (Samin et al., 2018).

Widely spread Palearctic species. Development takes place in similar conditions as the previous species, often both species coexist.

Distribution in Palearctic: Azerbaijan, Austria, Belgium, Bosnia and Herzegovina, Bulgaria, Belarus, Croatia, Russia (European Territory, Far East), Czech republic, Denmark, Estonia, Finland, France, Great Britain, Germany, Georgia, Hungary, Italy, Japan, Liechtenstein, Lithuania, Netherland, Norway, Poland, Portugal, Romania, Slovakia, Slovenia, Spain, Sweden, Switzerland, Ukraine, Serbia and Montenegro (Jelínek, 2008), Iran (Samin et al., 2018).

\section{${ }^{\star}$ Xylographus bostrichoides (Dufour, 1843) (fig.1, 6)}

Material examined: Golestan Prov., $11 \mathrm{~km} \mathrm{SE}$ of Minudasht, $37^{\circ} 10.151^{\prime} \mathrm{N} 55^{\circ} 28.101^{\prime} \mathrm{E}, 447 \mathrm{~m}$ amsl, 13-15.06.2015, 32 exx. leg. R. Królik et A. Lasoń from fungi on Populus sp. et Fagus orientalis, 49 exx. reared 20.VI-31.08.2015, cult. R. Królik (USMB, RKPC); Mazandaran Prov., Sisangan National Park, 36 35.211' N $51^{\circ} 48.103^{\prime}$ E, 8.06.2015, 32 exx. leg. T. Jaworski (RKPC).

Widespread Palearctic species, exception of extremely northern areas. Found most often on basidiomes of Fomes fomentarius, Ganoderma and Phellinus Quél.

Distribution in Palearctic: Algeria, Azerbaijan, Austria, Bosnia and Herzegovina, Belarus, Croatia, Cyprus, Czech Republic, France, Greece, Hungary, Italy, Morocco, Poland, Romania, Russia (South European Territory), Slovakia, Spain, Tajikistan, Turkey, Ukraine, (Jelínek, 2008). Iran (first record).

\section{Species for which the presence in Iran is doubtful or requires confirmation:}

Cis jacquemartii Mellié, 1849

Distribution: Azerbaijan, Austria, Bosnia and Herzegovina, Belarus, Croatia, Czech Republic, Denmark, Finland, France, Great Britain, Germany, Georgia, Greece, Hungary, Italy, Latvia, Norway, Poland, Romania, Russia (European Territory), Slovakia, Spain, Sweden, Switzerland, Turkey, Ukraine (Jelínek, 2008).

Form Iran reported by Samin et al. (2018 b). Due to the general distribution, we provide this data as doubtful. Evidence specimens needs verification, perhaps this is a wrong determination of C. lasoni. 
Cis lineatocribratus Mellié, 1849

Distribution: Azerbaijan, Austria, Bosnia and Herzegovina, Bulgaria, Belarus, Croatia, Czech Republic, Denmark, Estonia, Finland, France, Germany, Georgia, Hungary, Italy, Latvia, Liechtenstein, Norway, Poland, Romania, Serbia and Montenegro, Russia (North and Central European Territory), Slovakia, Sweden, Switzerland, Ukraine (Jelínek, 2008).

Form Iran reported by Samin et al. (2018 a). Due to the general distribution, we provide this data as doubtful. Evidence specimens needs verification, perhaps this is a wrong determination of C. matchanus.

Cis villosulus (Marsham, 1802)

Distribution: Austria, Belgium, Belarus, Croatia, Czech Republic, Denmark, France, Great Britain, Germany, Georgia, Greece, Hungary, Ireland, Italy, Liechtenstein, Netherland, Norway, Poland, Portugal, Russia (European Territory, East Siberia, Far East), Slovakia, Spain, Sweden, Switzerland, Turkey, Ukraine (Jelínek, 2008).

Form Iran reported by Samin et al. (2018 b). Due to the general distribution, we provide this data as doubtful. Evidence specimens needs verification, perhaps this is a wrong determination of C. rugulosus.

Octotemnus glabriculus (Gyllenhal, 1827)

Distribution: Azerbaijan, Austria, Belarus, Belgium, Bosnia and Herzegovina, Bulgaria, China (Liaoning), Croatia, Czech Republic, Denmark, Finland, France, Great Britain, Germany, Georgia, Hungary, Ireland, Italy, Japan, Latvia, Liechtenstein, Lithuania, Luxembourg, Macedonia, Netherland, Norway, Poland, Portugal, Romania, Russia (European Territory), Slovakia, Slovenia, Spain, Sweden, Switzerland, Turkey, Ukraine (Jelínek, 2008).

Form Iran reported by Samin et al. (2018 b). Due to the general distribution, we provide this data as doubtful. Evidence specimens needs verification, perhaps this is a wrong determination of $O$. rugosopunctatus.

\section{Check list of Ciidae of Iran}

CIIDAE Leach, 1819

Ciinae Leach, 1819

Orophiini C. G. Thomson, 1863

Octotemnus Mellié, 1847

O. rugosopunctatus Drogvalenko, 2002

Ropalodontus Mellié 1847

R. perrini Reitter, 1878

Xylographus Mellié, 1847

X. bostrichoides (Dufour, 1843)

Ciini Leach, 1819

Cis Latreille, 1796

C. boleti (Scopoli, 1763)

C. castaneus (Herbst, 1793) 
C. chinensis Lawrence, 1991

C. comptus Gyllenhal, 1827

C. fusciclavis Nyholm, 1953

C. lasoni Królik, 2016

C. lineatocribratus Mellié, 1849

C. matchanus Reitter, 1915

C. reitteri Lopes-Andrade, 2002

C. rugulosus Mellié, 1849

C. striatulus Mellié, 1849

C. submicans Abeille de Perrin, 1874

C. tomentosus Mellié, 1849

C. villosulus (Marsham, 1802)

Ennearthron Mellié, 1847

E. cornutum (Gyllenhal, 1827)

Strigocis Dury, 1917

S. bicornis (Mellié, 1849)

Sulcacis Dury, 1917

S. fronticornis (Panzer, 1805)

S. nitidus (Fabricius, 1792)

\section{Discussion}

This study was conducted in the North forest of Iran, which show high variation in plants and insects species (Sagheb Talebi et al., 2014, Müller et al., 2018). Although family Ciidae has not been specially studied in Iran, results of the current study reach the fauna of North Iran to represent 7 genera and 19 species. The most common species belongs to the genus Cis and first records in this study which found associated by fungi is negotiable. Further study is needed to confirm their accurate association. According to the comparison of bordered countries ciid fauna and high diversity of host plants in the Hyrcanian Forest of Iran, these records would not be a final number and obviously would increase in the future. According to the recent studies (Królik, 2016), the richest fauna of this family is in Azerbaijan and Georgia with 22 and 21 species, respectively. Ten species were reported in Turkey, but in Turkmenistan and Afghanistan only one species is recorded, due to the lack of forest and humid weather contitions (Jelínek, 2008; Królik, 2016). Since the forests of north Iran overall with the forest areas of Azerbaijan (the Talysh Mountains), where there are several additional species of Ciidae, we would expect to find more ciid species in the Hyrcanian Forest. However, Ciidae are one of the saproxylic beetles groups, which are important in wood decomposition, Therefore, fauna and biodiversity deserves further study in the future for successful conservation of the forest ecosystems.

Authors special thanks to Dr. Ehsan Torabi and Marjan Heidarian ( $\mathrm{PhD}$ graduated in Entomology, University of Tehran) for their help and thoughtful comments and the second author would like to thank his colleagues: Andrzej Lasoń and Tomasz Jaworski for handing the specimens for research.

\section{Disclosure Statement}

No potential conflict of interest was reported by the authors. 


\section{References}

Amini, S., Sheikhnejad, H., Hosseini, R. 2014. The first report of Cis multidentatus (Coleoptera: Ciidae) for the fauna of Iran. The 21th conference of Iranian Plant Protection Congress, At University of Urmia, Iran.

Amini, S., Sheykhnejad, H., Hosseini, R. 2015. First record of Cis chinensis (Coleoptera: Ciidae) from Iran. Journal of Entomological Research of Islamic Azad University, 8, 165-169 [In Persian.]

Diéguez Fernández, D., 2013. Coleoptera. Familia Ciidae. Algunas citas de cíidos de España. Arquivos Entomolóxicos, 8, 103-106.

Drogvalenko, A. N. 2002. New and rare species of beetles (Insecta: Coleoptera) for fauna of Ukraine. Information 2. Izvestiya Kharkovskogo Entomologicheskogo Obshchestva, 9, 9-19 [In Russian].

Graf-Peters, L. V., Lopes-Andrade, C., Silveira, R. M. B., Moura, L. A., Reck, M. A., Nogueira-de-Sá, F. 2011. Host Fungi and Feeding habits of Ciidae (Coleoptera) in a Subtropical Rainforest in Southern Brazil, with an overview of Host-Fungi of Neotropical Ciids. Florida Entomologist, 94, 553-566.

Jelínek, J. 2008. Family Ciidae Leach, 1819. In: Löbl, I. and Smetana, A., eds. Catalogue of Palaearctic Coleoptera, Vol. 5, Tenebrionoidea. Apollo Books, Stenstrup, 55-62.

Jonsell, M., Nordlander, G. 1995. Field attraction of Coleoptera to odors of the wood-decaying polypores Fomitopsis pinicola and Fomes fomentarius. Annales Zoologici Fennici, 32, 391-402.

Kawanabe, M. 1995. A synonymic note on Paraxestocis unicornis Miyatake (Coleoptera, Ciidae). Elytra, 23 (2), $175-176$.

Królik, R. 2016. Two new species and nomenclatural changes in the Cis castaneus species group (Coleoptera, Ciidae). Zootaxa, 4114, 492-500.

Królik, R., Szołtys, H., Melke, A. 2017. Ciidae (Coleoptera) Pogórza Przemyskiego. Rocznik Muzeum Górnoślaskiego w Bytomiu Przyroda, 23 (online 006), 1-13.

Lawrence, J. F. 1991. Three new Asiatic Ciidae (Coleoptera: Tenebrionoidea) asociated with commercial, dried fungi. Coleopterists Bulletin, 45, 286-292.

Lawrence, J. F. 2016. The Australian Ciidae (Coleoptera: Tenebrionoidea): A preliminary revision. Zootaxa, 41, 1-208.

Lawrence, J. F., Lopes-Andrade, C. 2010. Ciidae Leach in Samouelle, 1819 In: Leschen, R. A. B., Beutel, R. G., Lawrence, J. F., eds. Handbuch der Zoologie/Handbook of Zoology, Band/Volume IV Arthropoda: Insecta, Teilband/Part 38, Coleoptera, Beetles, Vol. 2, Morphology and Systematics (Polyphaga partim). Walter de Gruyter, Berlin, 504-514.

Lohse, G. A., 1967. Familie Cisidae. In: Freude, H., Harde, K. W. \& Lohse, G. A., eds. Die Käfer Mitteleuropas, Bd 7. Goecke Evers Verlag, Krefeld, 280-295.

Lohse, G. A., Reibnitz, J. 1991. Cis multidentatus (Pic), ein unbeachtetern Vorratsschädling (Coleoptera, Cisidae). Acta Coleopterologica, 7, 103-105.

Lopes-Andrade, C. 2008. The first record of Cis chinensis Lawrence from Brazil, with the delimitation of the Cis multidentatus species-group (Coleoptera: Ciidae). Zootaxa, 17 (55), 35-46.

Müller, J.,Varandi, H., Babaii, M., Farashiani, M., Sageb-Talebi, K., Lange, F., Gossner, M., Jarzabek-Müller, A., Roth, N., Thorn, S., Seibold, S. 2018. The diversity of saproxylic insects (Coleoptera, Heteroptera) on four tree species of the Hyrcanian forest in Iran. Journal of Insect Conservation, 22 (3-4), 607-625. https://doi. org/10.1007/s10841-018-0089-1

Orledge, G. M., Reynolds, S. E. 2005. Fungivore host-use groups from cluster analysis: patterns of utilization of fungal fruiting bodies by ciid beetles. Ecological Entomology, 30, 620-641.

Poussereau, J., Rose, O. 2010. Contribution á la connaissance des Ciidae de l'île de la Réunion (Coleoptera: Tenebrionoidea). Coleopteriste, 13, 30-31.

Reibnitz, J. 1999. Verbreitung und Lebensräume der Baumschwammfresser Südwestdeutschlands (Coleoptera: Cisidae). Mitteilungen Entomologischer Verein Stuttgart, 34, 1-76.

Reibnitz, J., Graf, R., Coray, A. 2013. Checklist of the Ciidae (Coleoptera) of Switzerland, with comments on nomenclature and ecology. Bulletin de la Société Entomologique Suisse, 86, 63-88.

Reibnitz, J., Kunz, W. 2011. Cis chinensis Lawrence, 1991 - neu für Baden-Württemberg (Col., Ciidae). Mitteilungen Entomologischer Verein Stuttgart, 46, 45-85.

Reitter, E. 1901. Byrrhidae (Anobiidae) und Cioidae. Bestimmungs-Tabelle der europäischen Coleopteren. XL07. (Heft 47). Brünn, Edm. Reitter, 1-64.

Rose, O. 2009. Cis chinensis Lawrence, 1991 nouveau pour la faune de France métropolitaine et celle de l'île de la Réunion (Coleoptera, Tenebrionoidea, Ciidae). L' Entomologiste, 65 (5), 281-284.

Sagheb Talebi, Kh., Sajedi, T., Pourhashemi, M. 2014. Forests of Iran: A Treasure from the Past, a Hope for the Future. Springer, Dordrecht, 1-152.

Samin, N., Bunalski, M., Kubisz, D., Háva, J., Navaeian, M., Otero, J. C., Hawkeswood, T. J., Zhou, H., Sakenin, H., Jędryczkowski, W. B. 2018. Contributions to the knowledge of the distribution of select Coleoptera families from Iran. Wiadomości Entomologiczne, 37 (4), 197-209. 
Shugran, A. H. M., Augul, R. S., Al-Khesraji, T. O., 2018. List of insects associated with macrofungi in Tikrit city, Salahadin governorate, Iraq. Bulletin of Iraq Natural History Museum, 15 (1), 41-55.

Souza-Gonçalves, I., Lopes-Andrade, C. 2018. The Cis multidentatus Species-Group (Coleoptera: Ciidae): New Species, New Records, Complementary Descriptions and an Identification Key. Annales Zoologici, 68, 501-518.

Souza-Gonçalves, I., Orsetti, A., Lopes-Andrade, C. 2018. Synopsis of Cis Latreille (Coleoptera: Ciidae) from southern Africa. Insects, 9, 184.

Received 3 January 2020

Accepted 25 August 2020 